\title{
Social Conditioning of Emotional Arousal - Psychosocial Need to Prevent Forensic Behavior
}

\author{
Mukundan CR* \\ Department of Neuropsychology, Bangalore, India
}

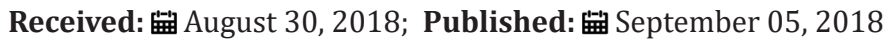

*Corresponding author: CR Mukundan, Department of Neuropsychology, Honorary Chairman, Axxonet Brain Research Laboratory, Bangalore 560029, India

\begin{abstract}
Emotional arousal has been considered provide the drive needed to propel a living being into responses and action. Responses are executed automatically as per the learnt method, whereas an action is executed as per a plan for achieving specific effects and goals. An action or response is automatically initiated when the drive reaches a Critical Level of Potentiation (CLP) [1]. Emotion is an internal arousal state needed for providing the drive required for the initiation of all responses and actions. Another important processing is the cognitive judgments of the inputs and output, which may mold the actions and responses into positive or negative emotional effects. The cognitive judgments of the behavioral responses and actions of others induced emotions in the receiver, which may be experienced as different levels of happiness, pleasure, pain, fear, sadness, and several more of such feelings, which may be specifically associated with the experiences of an individual. The experiential profile of the drive may generally constitute the positivity or negativity experientially associated with of the propelling force and what they may behaviorally accomplish in the self and others. Social conditioning helps everyone to build the drive stepwise manner, so that it may reach the CLP level gradually and one can control the responses and actions, so that the best and optimum effects are achieved. This learning method must be part of the social system, so that each growing child is trained to build their drive, which would initiate responses and actions.
\end{abstract}

Keywords: Emotional arousal; Critical Level of Potentiation; Bereitschafts potential; Virtual reality; Spiritual force; Preattentive emotional arousal; Cognitive judgments

\section{Introduction}

Living systems make physical contacts with realities through sensory-motor systems, which is experienced by the system. Experiencing is therefore the most vital method for making contacts with reality and reality verifications. A major experiential component is the interpretive process of the contacts, which helps to encode or transcode the experiences. The encoded and transcoded components of the sensory-motor contacts constitute the experience, which the system could reproduce as remembrance. Experiential verifications are the basic methods of subjective reality verifications, used by every individual and living being, which may also be influenced by the knowledge derived from similar previous experiences. Additional human method of verification is the scientific methods of verification, which do not need experiential substantiation as the verification is objective and beyond subjective experiences. Experiential verifications help to bring direct contacts with reality, though the verification is essentially subjective and is controlled by the needs and suggestive effects of the experiences, which are used for the interpretations and justifications of the experiences. On the other hand, verifications objectively carried out without consideration of their subjective experiential effects are considered scientific methods of verification, as they could repeated for eliciting the same results, by all even if they have their own experiential effects, and at any time and place. The same results could be repeated elicited by a system using specific experimentation, whether carried out by man or machine. Any change in the results could be attributed to specific changes in the methods of experimentation or additional effects acquired or employed by the system, all of which could be objectively evaluated and interpreted. On the other hand, experiential verification depends on the concepts used by the experiencing person, his or her mental and physical states, and the consequential effects that be generated by the experiencing person. Hence experiences may not be repeatedly elicited by the same person and others, who share different conceptual systems for explaining the changes and personal effects that may be noticed or experienced. Ancient 
methods were to employ subjective interpretive methods for assessing and explaining experiences, as they did not know how to employ objective scientific methods.

Scientific and experiential explanations and interpretations may therefore be diverse. However scientific experimentations and explanations may not always be possible, especially when one is not aware of the objective presence of variables and the changes that may occur, with different effects present controlling and contributing to those effects. On the other hand, experiential interpretations depend mainly on the knowledge base one uses, and the acceptance of the presence of different external factors, which one 'believes' to be present during such experiential generations. The need for personalized knowledge of such factors and their effects, which may not be objectively verifiable, render them special attributes, which could only be personally acquired, experienced, and understood. They are often labelled as spiritual effects, obtainable only through beliefs and mental attributes, which are not objectively controllable and measurable, and hence do not support scientific studies and explanations. The strongest need for spiritual thinking and experiences is the presence of belief in the strength of the force and the principle, which contribute to spiritual experiences. As spiritual force is considered essentially a supernatural one, its presence could be experienced only when one has faith in it and accept it as the force which contribute to all personal experiences. Spirituality is indeed a man-made experiential dimension, which could be understood only when one is prepared to accept and has faith in the experience and its explanations, though the spiritually engineered experiences are very strong and may have enormous experiential effects on the individual who has accepted its presence.

Most significant development of the human system is the functional growth of the brain, which has enabled it to learn methods to encode and transcode all sensory-motor contacts and create objectives for every action and movement in terms of specific goals, and to encode what we call purpose for living and finally to develop systems of life, which incorporate and serve the purposes of living. These abilities revolutionized human life as every individual born and growing considers that he or she has a purpose for living. Acceptance of such purposes by group of individuals help to create social systems, supporting and reinforcing such purposes and learning the art and methods of living in a system. Every newborn child during growth learns the art of living and the need to know and build a useful and purposeful life style for the self. This is strongly reinforced in many individuals by the development of a faith that these aims of life have been created by a higher force, who is also responsible for the creation and maintenance of the universe. This worked as a greater and stronger reinforcing concept in most of the individuals and in their social groups, though there are also those who have not entertained such concepts, as they have not believed in such universal controlling force. Living without dependence on such belief system is as old as the Vedic period. Using the brain functions, and creation of similar capabilities in external systems, man could create new realities, which could immensely enhance man's capabilities and comforts. The world they live have been undergoing drastic progressive changes all through years, because of the scientific inventions and changes man could make. The revolutionary changes made by man in living styles, comforts, methods for controlling the environment, own body and the brain and movements across the land and sea are some of those. The only capability that has not changed in the human being is the presence of emotional arousal, which has always served as the driving force of life. Emotions are still molded positively and negatively, as it was always done even thousands of years back. Use of virtual reality has also become a routine method of living [2]. As part of living, responses and actions are made to both physical and virtual realities.

A response is always evoked by a sensory signal, which may spell alarm, fear, or need for help, as responses are generally meant as automat reaction to threats from nature. The detection of threat or associated emotional arousal may take place at a subcortical level itself, though it may not be associated with regular perception and judgments. This has been called a "preattentive emotional response" [3-11], as it is a response initiated automatically, even without attending to it, which is normally followed by its perception - recognition, and transcoding or encoding contributing to awareness. The former is indeed a survival need, important for human beings and more for animals [12-14]. On the other hand, an action is initiated by a need state developed within the individual, and the need state may of current or later importance, or it may be initiated by the plans to create or develop a new system of functioning. Behavior is combination of responses and actions. Unexpected events occur to human beings, when they travel and move around, which could cause personal hurts and damages, unless they learn to avoid them in an automatic manner without their perception and intentional problem-solving efforts. The frontal subcortical areas associated with hippocampus, amygdala, etc. take care of the urgent and threatening needs to respond to external events which may become a survival threat are taken care of these areas by such automatic responses, which could take the system away from the threatening challenges. There is indeed a rise in the threatening condition and the associated emotional arousal. The sudden enhancement of emotional arousal may automatically initiate the responses which would help avoid threats and save oneself.

Studies of the brain mechanisms associated with initiation of movements have shown that motor activation necessary for the movement always start automatically, well before one becomes aware of the presence of initiation of the movement. This has been demonstrated by recording the Bereitschafts potential, which is directly associated with the neural activation needed for motor 
activities and movements [15-26]. The Bereitschafts potential which initiates the motor potentials starts about 2-3 seconds before the initiation of the motor potential in the motor system needed for the movement, when the individual is not aware of its presence $[28,29]$. The individual becomes aware of the movement only after the prior neural activation has already initiated the movement $[31,32]$. Therefore, if one wants to control any movement before it is initiated, one must have control on the drive, the rising of which to a critical point initiates the movement or the action. The simple parallel example is the process of heating water. When the water is heated and when it reaches 100 degrees centigrade, the water automatically starts boiling. In the same manner when the drive reaches a Critical Level of Potentiation (CLP), it initiates the movement automatically [1]. One must learn to control the drive early enough so that it does not reach the CLP point, when action would be automatically initiated. Learning to control and maintain the drive at a desired level must be acquired from infancy, and this is supported and made possible through social supports to the individual. Absence or reduced drive level also causes impediments to individuals as one would not be able to work for achieving goals, though one may be aware of the need to work for achieving goals of life. Drive and emotional arousal are different names used for the same arousal, which is an essential requirement for the initiation and completion of actions overcoming all impediments, if present. Additionally, it helps to anticipate or expect consequences and effects of one's own actions when one could learn to control style of carrying out the actions for obtaining the best or desired results.

What is important is to know that an action is initiated when the related drive reaches a critical limit $[1,14,27]$. Action is not initiated by a cognitive decision or cognitive state. Without drive or emotional arousal, no action is initiated, even if one has extensive cognitive appraisal of the need to act. Emotional arousal needs to be developed independently, as it does not develop by cognitive suggestions. Emotional arousal develops during and through physical actions and reactions with psychological involvement as in dancing, sporting, singing, praying, etc., all of which have various forms of sensory-motor presentations that we have developed, which could also cause emotional arousal states [19,20]. The emotional arousal and its molding are expected to be controlled from within by the ideal expectations and their controls of own sensory-motor contacts. One is not expected to mold own emotions based on the observed emotional states or reactions of other individuals (Sthita Prajna, cognitive molding of emotion- [33,34]). Cognitive processing that may take place during these reactions and movements help mold the emotional state into positive or negative emotions, which may highly differential effects on the self as well as on others.

One must learn to build the drive within and learn to maintain and utilize the drive in a controlled manner so that one engages only in socially approved and progressive activities. It is known that several of the criminal actions are carried out by individuals when they have no control on the associated drive, which propels them in such violent and undesirable directions. When the drive reaches the critical level, the system initiates and executes the actions, even if they know about its non-acceptability or criminal effects of the action. Social conditioning of emotional arousal is the only means to learn to keep the drive under control. One could learn this control on the drive since birth, from the first few months. The aim of the conditioning must be for the child to learn to wait, when necessary, for carrying out an act, at home and while being taken out. The child must learn to wait and carry out movements related to eating, drinking and playing in a controlled manner. Thus, a child should learn to carry out all movements and actions, considering the knowledge of possible reactions expected and present from others, and how it may merge with the needs and challenges of the specific surroundings. The child learns to carry out the movements and actions in a controlled manner while responding to others; and he does take care to see that he does not offend or hurt others. The child must also learn to think over as well as anticipate effects of his proposed actions and decide strategies for handling such effects and execute various actions. One could start exploring and engaging in adventurous activities as a child and master the self-controls needed for successfully carrying them out. He must learn to finally carry out an action considering all possible consequences and his own proposed reactions to them. Obviously, a child may take long periods for developing and learning a very appropriate positive action method. However, they often learn fast the immediate effects of carrying out negative actions and decide on the need to indulge in them for personal benefits and advantages.

\section{Drive vs Cognitive Processing Abilities}

There need not be a comparison between the drive level one employs and the cognitive abilities one may have. A high cognitive skill would enhance the quality of intellectual performance of an individual, provided he or she can utilize high drive levels for activities. A person with average intelligence or cognitive ability may reach high levels of achievement by utilizing high drive and working hard. The opposite results are frequently found in people with poor drive levels, even when they have high cognitive abilities. Most of those who indulge in uncontrolled activities, in the forms assault of women and other members of the society, are those who indulge in the said activity in uncontrolled manner as they get induced into it by raised emotional arousal or drive at that critical moment. They may later repent about their involvement and actions and want to be forgiven. In many countries, such assaults are very common, and occur in hundreds, indicating large scale absence of social conditioning of emotional arousal during the upbringing of their children. It may be considered an indication of the absence of effective social systems for controlling interactions of human behavior, which may reflect inadequacies precipitating violent social interactions at all levels [1]. These in turn reflect the absence 
of value systems which control social behavior and the absence of civilized norms for the control of social interactions among groups of people. Presence of social conditioning and social learning are very important strong indicators of civilized society, even if they may not have any spiritual biases in their styles of living.

Emotional arousal serves as the driving force for propelling the system to move and work in the form of responses and actions. Cognitive abilities and cognitive decisions would channelize these steps of responses and actions in a positive gainful manner to the individual. Cognitive judgments mold the emotional arousal in a positive or negative manner, fully or partly, or it may remain unmolded when the individual makes special efforts not to mold own emotional arousal using such judgments, as in "Sthita Prajna". Regulation of the drive is needed for controlled performance of responsive actions so that they do not cause damage or pain to the self and others and would facilitate forward movement and progressive actions. Social accommodation of other individuals must be a regulatory principle that allows propulsion oneself by emotional arousal in various situations. However, absence of drive, will not help the system to move forward or to higher levels of achievement. On the other hand, high drive will allow one to achieve high goals even if one cannot make highly progressive and original cognitive judgments, even by following the directions shown by someone else, who may be an expert in such decision making. What is important is the fact one cannot work and move forward without drive or emotional arousal. Criminal and violent activities are easily committed by those who have no control on their emotional drive. They indulge in such activities when the drive reaches the critical level of potentiation (CLP), as already pointed out. Absence of control on drive occurs, when it is not acquired during the developmental stages. Absence of control does not mean absence of drive. Drive is the life force, which propels every individual or living being to move around, respond and act. The drive is normally activated by physical-biological-psychological needs of survival and growth. The drive would propel responses and actions by each system as per the cognitive judgments of the needs for survival and growth, independently in a positive or negative manner or their combinations. The cognitive judgments induce molding effects on the drive, when it becomes specific emotions, which one learns to apply for controls as per the psychosocial and psychobiological needs one has identified from their resources created by the group of individuals over the past and in the present. The drive may necessitate the choice of initiating responses and or actions for continuous survival and growth.

Ancient as well as traditional practices have been to identify the drive as a spiritual force and label its presence as soul, consciousness, etc., which people considered to merge with a higher universal force of an absolute nature. We still consider the driving force as the life force and believe that it is the same which was earlier considered as the life force. Difficulty in its identification comes when it gets cognitively molded, when we label them as positive or negative emotions. In unmolded state, it is just the drive within the living system, which we still like to identify as the life force, or a force, which cannot be physically measured and hence amounts to be called a spiritual force. It is easily recognized as emotion, when cognitively molded, as one learns to mold it cognitively as per one's needs and the judgments one learns to make. When the same drive is molded into a positive or negative state, we normally call emotional state by specific names. However, one may preserve opportunities to experience the force without aggressive effects and label the state with peace and calmness as during meditation, praying, musical endeavors and performances with sensory-motor contacts like dancing, sports, and other artistic performances, with nil or minimum positive-negative emotional molding effects. In the normal course of development, each one learns to make cognitive judgments of the emotional arousal detected in the behavior of others, and automatically respond to them with what one has learnt to react and express with what one considers appropriate to safeguard interest of the self. Controls are applied by the parents and elders in the family and the society for inhibition and control of emotional arousal, instead of automatic initiation of behavior or responses by heightened emotional arousal, so that one carries out only socially accepted reactions. When one does not have such controls, emotional arousal rising to the critical limits (CLP) automatically initiates a related behavioral response, which may be violent, insulting, and destructive to another person, and which may need to be legally handled by the society.

Social conditioning practiced by the society members as per the accepted legal and social values of the society alone would help an individual to control the emotional arousal so that it does not rise to the critical limit when the action would be automatically initiated. Absence of such social conditioning encourages individuals to carry out responses as per the uncontrolled emotional arousal. Absence of practice of socially validated responses incites asocial behavior against persons of opposite sex, elderly, younger and disabled individuals, which are extensively presently in several societies, where they do not practice social conditioning among their younger and growing children, as they do not get the privilege of controlling effects of social conditioning. Several hundreds of such cases occur in several of our societies and in their social organizations, where social controls are not practiced by individuals on their emotional arousal, and negative responses and actions easily and automatically occur among their members. Religious values and beliefs may help develop the control over emotional arousal in people. They develop normal self-controlling capability only when they learn to control emotional arousal as young individuals, in various social practices and thereby master the necessary emotional controls, so that negative emotional arousal and associated aggressive responses are not automatically initiated. Presence of self-control helps one to exercise controls on the emotional drive, which helps 
to control and all-together stop negative actions and responses Limited number of societies exercise social conditioning for selfcontrols of individuals, whereas there are societies with absence of social conditioning and presence of large number of violent actions, especially against women and elderly. Social conditioning works on even animals, provided they are given adequate training. Absence of social conditioning among human beings ruins all their civilized and controlled behavior, replacing them by selfish behavior which create violent and criminal effects. There is no place where controlled social behavior is not needed. Walking and riding on the roads, entering a vehicle, finding a seat for oneself inside a hall, room, or a vehicle, and sharing food and any other item, which others too need, all need sharing with other individual, which one could learn only if one is aware and respect the needs and rights of other individuals. Allowing rising of own emotional arousal is indeed a positive and creative effort provided one could experience and utilize the emotional arousal for initiating and performing creative actions, which could enhance personal achievements. Social conditioning is the process of learning to control own emotional arousal so that negative reactions which may automatically be initiated against others could be controlled by everyone when emotional arousal reaches the critical limits.

Drive is a life force, which is not merely the presence of calories of energy or its potential strength. We have not been able to find a comparison with any other aspect of body or body function. Emotional energy is a life force, a mental (?) energy and not merely a physical energy. That emotional arousal cannot be compared with any other physical energy, has made it necessary to associate a unique life force with it, which we have done by calling it a spiritual force or soul of life, etc., with which we could not compare any other physical forces in the universe. It is a descriptive statement suggesting a force, without which no responses or actions are possible with the life system. The responses and actions of a living system are different from the responses elicited at the physical material levels. It is because of this nonmaterialistic nature of the emotional arousal, it is identified as a nonmaterial - spiritual force.

\section{References}

1. Mukundan CR, Ajayan P, Kacker P, Chetan SM, Vyas JM (2014) Violent Behavior: Absence of Social Conditioning of Drives during Neurodevelopmental Stages. International Journal of Indian Psychology 2(1): 1-33.

2. Mukundan CR (2018) Understanding and dealing with the mental creations: living in real and virtual worlds. J Psychol Clin Psychiatry $9(4): 394-398$.

3. De Gelder B (2005) "Unconscious fear influences emotional awareness of faces and voices". Proceedings of National Academy of Sciences, U.S.A. 102(51): 18682-18687.

4. De Gelder B (1999) "Non-conscious recognition of affect in the absence of striate cortex". Neuro report 10(18): 3759-3763.

5. De Gelder B, Rouw R (2000) “Paradoxical configuration effects for faces and objects in prosopagnosia". Neuropsychologia 38(9): 1271-1279.
6. Bishop SJ (2004) "State anxiety modulation of the amygdala response to unattended threat-related stimuli". Journal of Neuroscience 24(46): 10364-10368.

7. Morris JS (1998) "Conscious and unconscious emotional learning in the human amygdala". Nature, 393(6684): 467-470.

8. Whalen PJ (1998) “Masked presentations of emotional facial expressions modulate amygdala activity without explicit knowledge". Journal of Neuroscience 18(1): 411-18.

9. Soares JJ, Ohman A (1993) "Preattentive processing, preparedness and phobias: Effects of instruction on conditioned electrodermal responses to masked and non-masked fear-relevant stimuli". Behavior Research and Therapy 31(1): 87-95.

10. Soares JJ, Ohman A (1993) "Backward masking and skin conductance responses after conditioning to nonfeared but fear-relevant stimuli in fearful subjects". Psychophysiology 30(5): 460-466.

11. Windmann S, Kruger T (1998) "Subconscious detection of threat as reflected by an enhanced response bias". Conscious Cognition 7(4): 60333.

12. Mukundan CR (2007) Brain Experience: Neuroexperiential Perspectives of Brain-Mind. Atlantic Publishers, New Delhi.

13. Mukundan CR (2015) "Brain at Work: Neuroexperiential Perspectives", Atlantic Publishers, New Delhi.

14. Mukundan CR (2017) "Emotion - The Driving Force", Red Shine Publication, ISBN 978-81-933277.

15. Shibasaki H, Barrett G, Halliday E, Halliday AM (1980a) Components of the movement-related cortical potentials and their scalp topography. Electroencephalography \& Clinical Neurophysiology 49(3-4): 213-226.

16. Shibasaki H, Barrett G, Halliday E, Halliday AM (1980b) Cortical potentials following voluntary and passive finger movements. Electroencephalography \& Clinical Neurophysiology 50(3-4): 201-213.

17. Shibasaki H, Barrett G, Halliday E, Halliday AM (1981) Cortical potentials associated with voluntary foot movements in man. Electroencephalography \& Clinical Neurophysiology 52(6): 507-516.

18. Libet B, Gleason CA, Wright EW Jr, Pearl DK (1983) Time of conscious intention to act in relation to onset of cerebral activity (readiness potential). Brain 106: 623-642.

19. Libet B (1978) Neuronal vs. subjective timing, for a conscious sensory experience. In PA Buser, A Rougeul-Buser (Eds.), Cerebral Correlates of Conscious Experience, Amsterdam/New York: North Holland. pp. 149162.

20. Libet B (1999) Do we have free will? Journal of Consciousness Studies 6: 47-57.

21. Libet B (2001) Consciousness, free action and the brain. Journal of Consciousness Studies 8: 59-65.

22. Haber SN, Kunishio K, Mizobuchi M, Lynd-Balta E (1995) The orbital and medial prefrontal circuit through the primate basal ganglia. Journal of Neuroscience 15 (7): 4851-4867.

23. Deecke L, Kornhuber HH (1978) An electrical sign of participation of the mesial supplementary motor cortex in human voluntary finger movement. Brain Research 159(2): 473-476.

24. Deecke L (1987) Bereitschaftspotential as an indicator of movement preparation in supplementary motor area and motor cortex. Ciba Found Symp 132: 231-250.

25. Deecke L (2000) The Bereitschaftspotential as an electrophysiological tool for studying the cortical organization of human voluntary action. Suppl Clin Neurophysiol 53: 199-206.

26. Libet B (1985) Unconscious cerebral initiative and the role of conscious will in voluntary action, The Behavioral and Brain Sciences, with open 
peer commentary and Libet's reply, Theory and evidence relating cerebral processes to conscious will 8: 529-566.

27. Mukundan CR, J Singh, Ray R, Desai N (1986) Bereitschaftspotential in alcoholics. Biological Psychiatry 21(11): 1090-1092.

28. Khanna S, Mukundan CR, Channabasavanna SM (1989) Bereitschaftspotential in melancholic depression. Biological Psychiatry 26(5): 526-529.

29. Madhavi R (1999) "Slow DC Potentials and the Study of Volition". Ph.D. thesis submitted to National Institute of Mental Health \& Neuro Sciences, Deemed University, Bangalore.

30. Matsuhashi M, Hallett M (2008) The timing of the conscious intention to move. European Journal of Neuroscience 28(11): 2344-2351.

To Submit Your Article Click Here: Submit Article

DOI: $10.32474 /$ PRJFGS.2018.02.000132

31. Soon CS, Brass M, Heinze HJ, Haynes JD (2008) Unconscious determinants of free decisions in the human brain. Nature Neuroscience 11(5): 543545 .

32. Mukundan CR, Arun Sasidharan, Priyanka Kacker (2017) "Sthita Prajna: A State to Control Cognitive Molding of Emotional Arousal". In $1^{\text {st }}($ Edn), Jitendra Mohan, Emerging Contours of Excellence, Publication Bureau, Punjab University, Chandigarh pp. 24-40.

33. Mukundan CR, Kacker P (2018) Molding emotion while cognitively processing physical \& virtual realities. EC Neurology 10(5): 354-366.

34. Mukundan CR, Kacker P (2018) Emotional Arousal - the Driving Force of Life. Journal of Psychology \& Clinical Psychiatry 9(3): 1 -12.

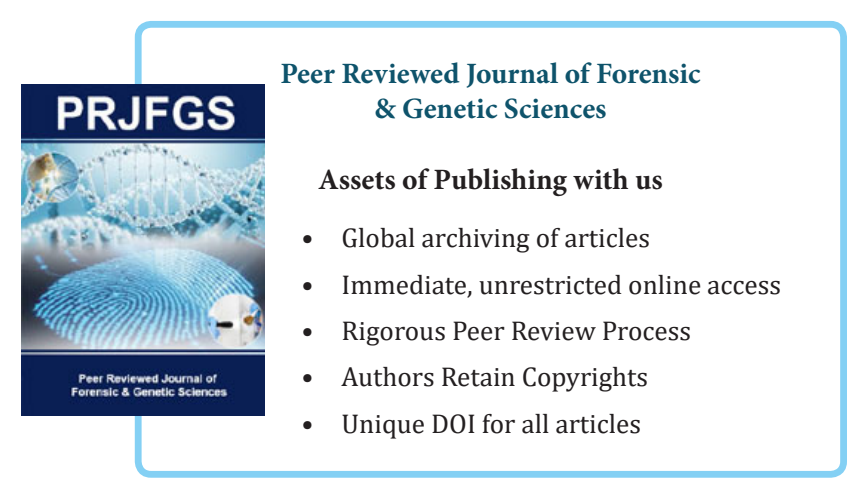

